\title{
Evaluation of Diagnostic Accuracy of Magnetic Resonance Imaging in Posterior Ligamentum Complex Injury of Thoracolumbar Spine
}

\author{
Gaurav Mehta ${ }^{1}$, Utsav Chandrashekar Shetty ${ }^{1}$, Dharamraj Meena ${ }^{2}$, \\ Ashok Kumar Tiwari ${ }^{1}$, Kishan Gopal Nama ${ }^{1}$, Dharmendra Aseri ${ }^{2}$ \\ ${ }^{1}$ Department of Orthopaedics, Government Medical College, Kota, India \\ ${ }^{2}$ Department of Radiology, Government Medical College, Kota, India
}

Study Design: Prospective diagnostic imaging study.

Purpose: The stability of the thoracic and lumbar spine depends significantly on the posterior ligamentum complex (PLC). Therefore, it is essential to diagnose PLC injuries accurately before deciding on a treatment plan for thoracolumbar injury patients. However, the efficacy of magnetic resonance imaging (MRI) in diagnosing PLC remains undetermined.

Overview of Literature: MRI has become the ultimate tool in diagnosing spine injury cases, as previous literature suggests that it has very high sensitivity and specificity. But this is still controversial and as many surgeons rely on just MRI for selecting the patient for surgery, it becomes important to know the diagnostic accuracy of it.

Methods: Patients who sustained injuries from T1 to L3 and required posterior surgery were prospectively studied. The treating surgeon and musculoskeletal radiologist participating in the study reviewed preoperative MRI images to characterize the level(s) of injury and the integrity of the six components of the PLC. These were classified as intact, incompletely disrupted, or disrupted. During the surgical procedure, the surgeon also classified each component of the PLC, and the radiologist's and surgeon's findings were compared.

Results: Out of 66 patients, 46 were males (69.7\%) and 20 were females (30.3\%), and the average age was 34.12 years. According to the kappa score, there was a moderate level of agreement between the radiologist's interpretation and the intraoperative findings for all PLC components except for the thoracolumbar fascia and ligamentum flavum for which there was a slight agreement. The sensitivity for the intact PLC components ranged from 100\% (supraspinous ligament) to $66.67 \%$ (ligamentum flavum). The specificity ranged from $100 \%$ (interspinous ligament) to $52 \%$ (thoracolumbar fascia). The Spearman's rank correlation ranged from 0.061 for the thoracolumbar fascia to 0.918 for the interspinous ligament, and the percentage agreement ranged from $81.82 \%$ (interspinous ligament to $36.36 \%$ (thoracolumbar fascia).

Conclusions: The sensitivity and specificity of MRI for diagnosing injury of the PLC in this study were lower than those previously reported in the literature. The integrity of the PLC as determined by MRI should not be used in isolation to determine treatment.

Keywords: Posterior ligamentum complex; Thoracolumbar injury; Spine; Magnetic resonance imaging; Diagnostic accuracy

Received Jan 20, 2020; Revised Mar 25, 2020; Accepted Apr 20, 2020

Corresponding author: Utsav Chandrashekar Shetty

Department of Orthopaedics, Government Medical College, Kota, Rajasthan 324005, India

Tel: +91-7829416781, E-mail: shetty.utsav@gmail.com 


\section{Introduction}

The diagnostic accuracy of spinal injury cases has improved in recent decades because of the advent of modern imaging techniques. Magnetic resonance imaging (MRI) has been an important diagnostic modality since it helps in deciding between conservative and operative management.

Spinal stability was defined by White and Panjabi [1] as the ability of the spinal elements to prevent the development of neurological injury and progressive deformity in the face of normal physiological processes; however, the identification of an unstable spine can be challenging [2]. The stability of the thoracolumbar spine is determined by the osseous ligamentous complex [3-6]. According to a meta-analysis conducted by Lee et al. [3] and Vaccaro et al. [2], the posterior ligamentum complex (PLC) makes a significant contribution to spinal stability. The PLC consists of the supraspinous and interspinous ligaments, the facet joint capsules, ligamentum flavum, and thoracodorsal fascia [4,6-9]. The Thoracolumbar Injury Classification System (TLICS) scoring developed by Vaccaro et al. [6] cites the integrity of the PLC as one of the important determinants when deciding the management approach for spinal injuries.

In a case of a grossly displaced or dislocated spine, the incompetence of the PLC is obvious. The members of the Spine Trauma Study Group identified plain radiographic signs as the most important determinant of PLC injury [3]; however, in the absence of obvious radiographic or clinical indicators, evaluating the integrity of the PLC can be difficult.

Current MRI technology includes the use of specific sequences to identify soft tissue injury. The fat saturation $\mathrm{T} 2$-weighted sequence is said to be the most reliable imaging modality in delineating the degree of PLC injuries [3]. However, the degree to which the MRI findings correlate with the actual tissue disruption is poorly understood. As treatment decisions for spinal injury are dependent on the integrity of the PLC, it is important to verify the diagnostic accuracy of MRI in detecting PLC injuries.

In this study, we aimed to evaluate the sensitivity, specificity, negative predictive value (NPV), positive predictive value (PPV), and diagnostic accuracy of MRI in PLC injury by comparing it with intraoperative findings.

\section{Materials and Methods}

This was a prospective cross-sectional study conducted at Goverment Medical College and Attached Group of Hospitals, Kota, Rajasthan from June 2017 to October 2019. We obtained approval from the institutional ethical committee (IRB approval no., F3/Acad-II/Plan/2019/1262). After providing written informed consent, patients with thoracolumbar spinal trauma were included in our study. We excluded patients who had pathological fractures.

First, we obtained an anteroposterior and lateral skiagram, a computed tomography (CT) scan, and the MRI of the patients. The MRI was done by fat saturation T2weighted sequence for an accurate delineation of the PLC injury [3]. Basic demographic data of patients and the type of fracture pattern were noted [10]. The participating radiologist reviewed the MRI to characterize the level of injury and integrity of six components of the PLC. If multiple spinal levels were involved, all levels within the surgical approach were analyzed.

On preoperative MRI, each component of the PLC was described as either intact, incompletely disrupted, or completely disrupted. Intact was defined as no visible change in the MRI signal (Figs. 1, 2); disruption was defined as clear MRI signal change with evidence of full discontinuity; and incomplete disruption was a diagnosis of exclusion, with the PLC structure of interest meeting neither the definition of intact nor that of disruption (i.e., visible

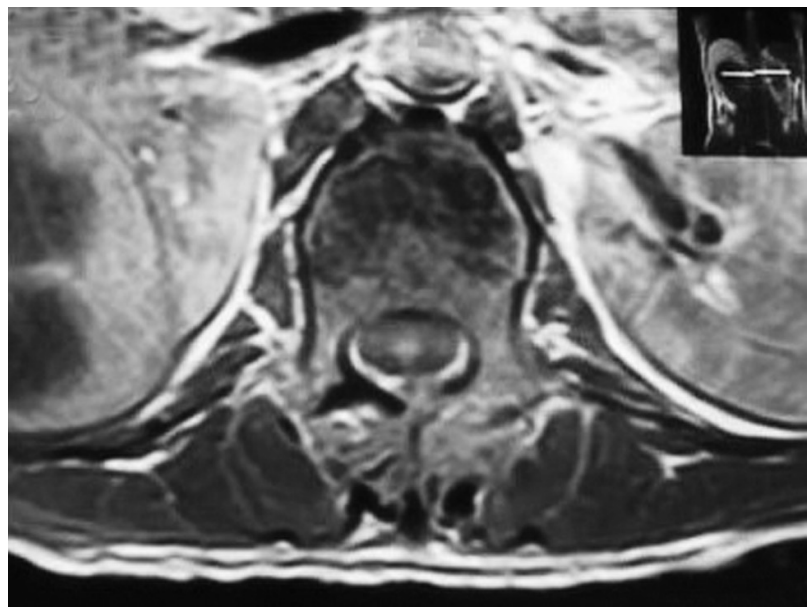

Fig. 1. Axial T1-weighted image of a 32-year-old male patient who sustained a compression fracture of the L1 vertebra in a road traffic accident with bilateral lamina fracture, disruption of the ligamentum flavum, intact left facet joint capsule, and thoracodorsal fascia. Because the right facet joint capsule cannot be identified with certainty as intact or disrupted, it is characterized as incompletely disrupted. 


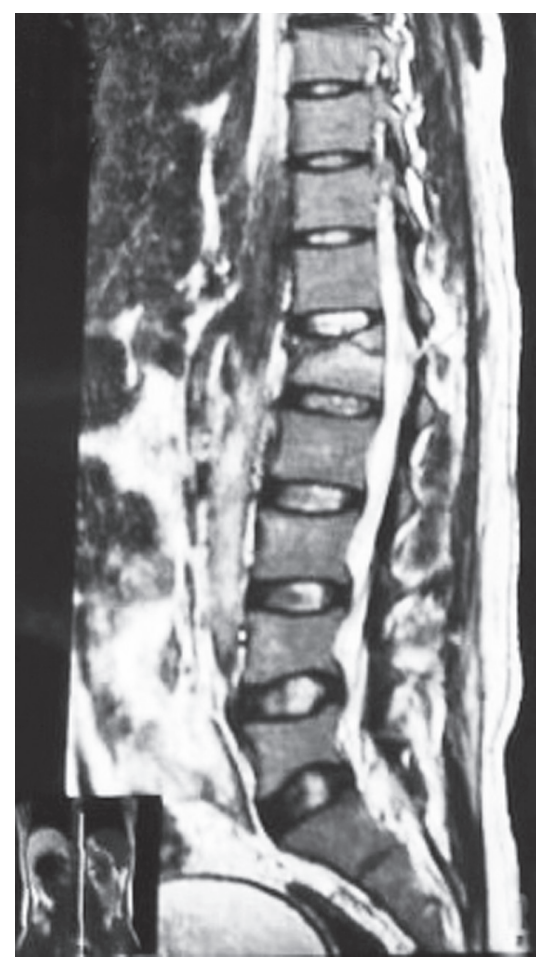

Fig. 2. Sagittal T2-weighted image of a 32-year-old male patient who sustained a compression fracture of the L1 vertebra in a road traffic accident with disruption of the ligamentum flavum and interspinous and supraspinous ligaments.

change in the MRI signal but failure to identify full discontinuity) [11]. The radiologist's findings were recorded, but the surgeon was blinded to them. The decision on surgery was based on the TLICS devised by Vaccaro et al. [2].

During surgery, the primary surgeon visually identified and assessed the integrity of the six components of the PLC. Each component was similarly classified by the surgeon. Intact was defined as the lack of visible injury or disruption; disruption was defined as the presence of visible injury with complete disruption as shown by the surgeon's ability to pass an instrument through the structure; and incomplete disruption was a diagnosis of exclusion, with the PLC structure of interest meeting neither the definition of intact nor that of disruption (Fig. 3) [11]. All intraoperative findings of the chief surgeon were witnessed and verified by the first assistant surgeon and recorded on a standardized data sheet.

The variables were summarized using frequency distribution and mean \pm standard deviation. The correlation between ordinal data was calculated using Spearman's rank-order correlation. The qualitative variables were expressed as contingency tables (intraoperative versus MRI findings), and their agreement was assessed using Cohen's

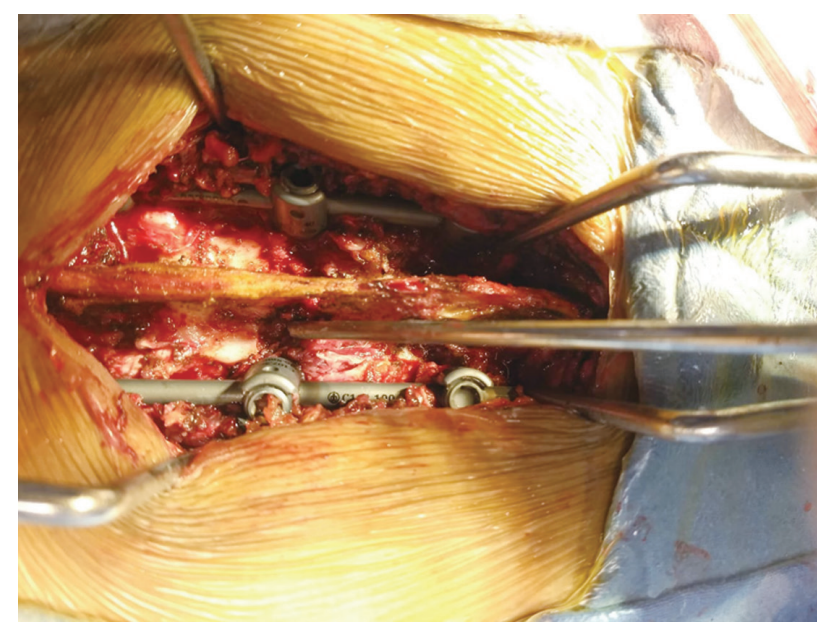

Fig. 3. Intraoperative image of the patient, surgeon assessing the status of the patient.

kappa statistics. Further, sensitivity, specificity, PPV, and NPV were also calculated. A $p$-value of $<0.05$ was considered statistically significant. Statistical analysis was performed using "R" programming language (The R Foundation for Statistical Computing, Vienna, Austria; https:// www.r-project.org/). Spearman's rank-order correlation coefficient can range from 0 to 1 , with 0 representing no correlation and 1 representing perfect correlation. A kappa score from 0.00 to 0.20 represents slight agreement, from 0.21 to 0.40 represents fair agreement, from 0.41 to 0.60 represents moderate agreement, from 0.61 to 0.80 represents substantial agreement, and above 0.8 represents near-perfect agreement. The percentage agreement between the radiologist's report and intraoperative findings, as well as the sensitivity, specificity, PPV, and NPV of MRI as predictors for the extent of injury, was calculated for each component of the PLC. Disruption and incomplete disruption categories of PLC injury were collapsed into a single category to calculate these measures.

\section{Results}

Fifty-eight patients who met the inclusion criteria were enrolled. The average age was 34.12 years (range, 20-54 years). There were 42 male and 16 female patients. There were 66 injuries, and the modes of injury mainly consisted of motor vehicle accidents comprising of 32 patients followed by fall from height (21 patients), fall of weight (three patients), and pedestrian accidents (two patients) (Table 1). The fracture morphology was classified as compression $(n=42)$, translation/rotation $(n=9)$, and distraction $(n=7)$ 
Table 1. Depicting the mode of injury in our study patients

\begin{tabular}{llc} 
Serial no. & \multicolumn{1}{c}{ Mode of injury } & No of patients $(\%)$ \\
\hline 1 & Motor vehicle accident & $32(55)$ \\
2 & Fall from height & $21(36)$ \\
3 & Fall of weight & $3(6)$ \\
4 & Pedestrian accident & $2(3)$ \\
\hline
\end{tabular}

Table 2. Morphology/pattern of the fracture

\begin{tabular}{lc} 
Pattern & No. of patients $(\%)$ \\
Compression & $42(72)$ \\
Translation/rotation & $9(15)$ \\
\hline Distraction & $7(12)$ \\
\hline
\end{tabular}

Table 3. Level of injury (including multiple level injuries)

\begin{tabular}{lc} 
Level & No. of patients (\%) \\
\hline Thoracic (T1-T11) & $8(12)$ \\
Lumbar (L2-L5) & $28(42.5)$ \\
\hline Thoracolumbar (T12, L1) & $30(45.5)$ \\
\hline
\end{tabular}

as described in Table 2. Most of the fractures were in the lumbar region followed by thoracolumbar, and the fewest were in the thoracic region (Table 3 ). There were eight patients with two-level injuries that were within the operating level.

Table 4 shows the frequency of disruption of each component of the PLC according to the radiologist's interpretation of the preoperative MRI and the intraoperative interpretation of the surgeon. Table 5 depicts the Spearman's rank-order correlation and kappa score comparison between the radiologist's and the surgeon's findings. The two scores were calculated considering only two categories, undisrupted and disrupted (including partially disrupted). The sensitivity, specificity, PPVs, and NPVs of MRI as an indicator of disruption of the various components of the PLC are reported in Table 6 separately for intact, partially disrupted, and completely disrupted components.

According to the kappa score, there was a moderate level of agreement between the radiologist's interpretation and the surgeon's intraoperative findings for all components of the PLC except for the ligamentum flavum and thoracolumbar fascia, for which there was a fair and slight

Table 4. Number of patients status in radiologists and surgeons observation

\begin{tabular}{|c|c|c|c|c|c|c|c|}
\hline \multirow{2}{*}{ Serial no. } & \multirow{2}{*}{ Ligament } & \multicolumn{2}{|c|}{ Intact } & \multicolumn{2}{|c|}{ Incompletely disrupted } & \multicolumn{2}{|c|}{ Completely disrupted } \\
\hline & & Radiologically & Intraoperatively & Radiologically & Intraoperatively & Radiologically & Intraoperatively \\
\hline 1 & Supraspinous & $44(67)$ & $34(52)$ & $10(14)$ & $6(10)$ & $12(19)$ & $26(39)$ \\
\hline 2 & Interspinous & $42(62)$ & $44(66)$ & $14(24)$ & $10(14)$ & $10(14)$ & $12(19)$ \\
\hline 3 & Ligamentum flavum & $34(52)$ & $36(57)$ & $12(19)$ & $4(5)$ & $20(29)$ & $26(39)$ \\
\hline 4 & Left facet capsule & $28(43)$ & $28(43)$ & $12(19)$ & $6(10)$ & $26(39)$ & $32(47)$ \\
\hline 5 & Right facet capsule & $26(39)$ & $26(39)$ & $14(23)$ & $10(14)$ & $26(39)$ & $30(46)$ \\
\hline 6 & Thoracodorsal fascia & $36(57)$ & $20(29)$ & $20(29)$ & $10(14)$ & $10(14)$ & $36(57)$ \\
\hline
\end{tabular}

Values are presented as number (\%).

Table 5. Depicts the Spearman's rank order correlation and kappa score comparison between the two considering the partially disrupted component to be disrupted for deducing it

\begin{tabular}{lcccc}
\hline PLC components & Spearman correlation & Kappa coefficient & p-value & Agreement (\%) \\
\hline Supraspinous & 0.648 & 0.523 & $<0.001$ & 72.53 \\
Interspinous & 0.918 & 0.648 & $<0.001$ & 81.82 \\
\hline Ligamentum flavum & 0.358 & 0.228 & 0.041 & 54.55 \\
Left facet capsule & 0.567 & 0.505 & 0.001 & 69.70 \\
\hline Right facet capsule & 0.664 & 0.57 & $<0.001$ & 72.73 \\
Thoracodorsal fascia & 0.061 & 0.099 & 0.735 & 36.36 \\
\hline
\end{tabular}

$p$-value and \% agreement are also shown in the table.

PLC, posterior ligamentum complex. 
agreement, respectively. The Spearman's rank correlation coefficient ranged from 0.918 (interspinous ligament) to 0.061 (thoracolumbar fascia).

\section{Discussion}

Spinal mechanical stability is primarily determined by the PLC according to studies conducted by James et al. [12] and Oxland et al. [13]. Vaccaro et al. [2] recently described the TLICS that consists of three primary axes that are independently important but complementary with each other for the characterization and treatment of fracture patterns, namely, (1) injury morphology, (2) integrity of PLC, and (3) neurological status. For appropriate classification and scoring according to TLICS, it is important to accurately diagnose the injury status of the various components of the PLC.

PLC disruption is considered to be one of the important criteria for recognizing an unstable injury, according to studies by Lee et al. [3] and Vaccaro et al. [2]. PLC injury status can be diagnosed by clinical examination, plain radiography, or CT scan when there are obvious indirect signs of PLC disruption like a palpable step-off or a defect detected on clinical examination and presence of splaying of the spinous process on plain radiography or CT, but the diagnostic accuracy of these signs is considered to be quite low as demonstrated by Lee et al. [3]. Others have suggested that MRI is a superior diagnostic tool for detecting PLC injury compared with other investigations $[14,15]$.

Almost all previous studies have suggested a good intraoperative correlation of MRI findings for PLC injuries apart from a study conducted by Vaccaro et al. [2]. Haba et al. [16] had concluded that the sensitivity and specificity of MRI in diagnosing supraspinous and interspinous ligament injury was $90.5 \%$ and $94.3 \%$, respectively, with a near-perfect agreement according to the kappa score, thus concluding it to be an important tool for diagnosing PLC injury and, therefore, an unstable spine. They found that T1-weighted MRI was superior to T2-weighted MRI for detecting supraspinous and interspinous ligament injuries. This study, being retrospective, relied on recorded surgical descriptions of anatomical injury. The diagnostic accuracy was investigated only for the supraspinous and interspinous ligaments where the PLC comprised six com-

Table 6. The sensitivity, specificity, PPV, NPV for magnetic resonance imaging as an indicator of disruption of the various components of the PLC

\begin{tabular}{|c|c|c|c|c|c|}
\hline PLC components & Status & Sensitivity & Specificity & PPV & NPV \\
\hline \multirow[t]{3}{*}{ Supraspinous } & Intact & 100.00 & 68.75 & 77.27 & 100.00 \\
\hline & Partially disrupted & 66.67 & 90.00 & 40.00 & 96.43 \\
\hline & Completely disrupted & 38.46 & 95.00 & 83.33 & 70.37 \\
\hline \multirow[t]{3}{*}{ Interspinous } & Intact & 95.45 & 100.00 & 100.00 & 91.67 \\
\hline & Partially disrupted & 60.00 & 85.71 & 42.86 & 92.31 \\
\hline & Completely disrupted & 50.00 & 92.59 & 60.00 & 89.29 \\
\hline \multirow[t]{3}{*}{ Ligamentum flavum } & Intact & 66.67 & 66.67 & 70.59 & 62.50 \\
\hline & Partially disrupted & 0 & 80.65 & 0 & 92.59 \\
\hline & Completely disrupted & 46.15 & 80.00 & 60.00 & 69.57 \\
\hline \multirow[t]{3}{*}{ Left facet capsule } & Intact & 71.43 & 78.95 & 71.43 & 78.95 \\
\hline & Partially disrupted & 66.67 & 86.67 & 33.33 & 96.30 \\
\hline & Completely disrupted & 68.75 & 88.24 & 84.62 & 75.00 \\
\hline \multirow[t]{3}{*}{ Right facet capsule } & Intact & 84.62 & 90.00 & 84.62 & 90.00 \\
\hline & Partially disrupted & 60.00 & 85.71 & 42.86 & 92.31 \\
\hline & Completely disrupted & 66.67 & 83.33 & 76.92 & 75.00 \\
\hline \multirow[t]{3}{*}{ Thoracodorsal fascia } & Intact & 70.00 & 52.17 & 38.89 & 80.00 \\
\hline & Partially disrupted & 60.00 & 75.00 & 30.00 & 91.30 \\
\hline & Completely disrupted & 11.11 & 80.00 & 40.00 & 42.86 \\
\hline
\end{tabular}

Values are presented as \%

PPV, positive predictive value; NPV, negative predictive value; PLC, posterior ligamentum complex. 
ponents. However, we used T2-weighted imaging, since it has been observed to have better sensitivity and specificity for assessing the integrity of all the components of PLC according to Lee et al. [3].

In a prospective evaluation of 34 patients conducted by Lee et al. [3] to evaluate PLC injury with MRI, plain radiography and clinical examination showed that there was no correlation between the clinical findings, plain radiographic findings, and intraoperative findings, although a significant correlation existed between the MRI and intraoperative findings with a sensitivity of $92.9 \%, 100 \%$, and $85.7 \%$ in detecting injury to the supraspinous ligament, interspinous ligament, and ligamentum flavum, respectively. Their analysis focused on only the three components leaving the facet joint capsules and thoracolumbar fascia [11].

Vaccaro et al. [11] showed a moderate level of agreement between a spinal radiologist's interpretation of MRI and the surgeon's intraoperative findings. They concluded that the specificity of MRI for diagnosing injury to the PLC was lower than the previously reported literature. The results ranged from a specificity of 53\% (thoracolumbar fascia) to $65 \%$ (ligamentum flavum) and a sensitivity of $79 \%$ (left facet joint capsule) to $90 \%$ (interspinous ligament), which were both lower than in the other studies. This relatively low specificity suggests that the MRI has a high false positive rate for detecting PLC injury. However, they had combined the sensitivity of MRI for detecting partially and completely disrupted components. Unlike them, we assessed the status of the PLC separately for detecting the sensitivity, specificity, NPV, and PPV for intact, partially disrupted, and completely disrupted components. Our study's MRIs were interpreted by a general radiologist, considering the lower availability of spinal radiologists in general practice rather than a dedicated spinal radiologist as was the case in the study by Vaccaro et al. [11]. Our study reported a fair-to-moderate agreement between a general radiologist's interpretation of MRI and a surgeon's intraoperative findings. There was a wide range of sensitivity, specificity, NPV, and PPV in detecting the status of PLC.

The results of our study were in accordance with the findings of Vaccaro et al. [11], but we observed a lower agreement between the MRI and intraoperative findings probably owing to the absence of a dedicated spinal radiologist in our study.

Our study has shown that the diagnosis of PLC injury based on preoperative MRI alone as interpreted by a radiologist, has a fair-to-moderate level of agreement with what is observed intraoperatively. The results of this study suggest that the diagnostic accuracy of MRI is not as high as suggested by previous studies. We observed that there is a high sensitivity but low specificity for diagnosing the PLC component injury separately as intact or disrupted as defined by Vaccaro et al. [11]. We believe that there is a need for better criteria for the evaluation of PLC disruption on MRI, and the current imaging protocols for thoracolumbar injury also need to be reconsidered.

The limitations of this study include the relatively small number of patients. A similar study on a larger scale is needed to eliminate confounding factors. Further, we only included patients who required surgical treatment via a posterior approach; therefore, patients requiring an anterior approach with subtle PLC injuries could not be accessed on MRI.

\section{Conclusions}

We conclude that the status of the PLC cannot be predicted with a high sensitivity and specificity by MRI contrary to earlier studies. If a surgical plan is based on MRI findings, there may be a tendency to operate unnecessarily on patients who have an intact PLC. Thus, MRI should always be coupled with other diagnostic modalities to determine the status of the PLC. Apart from the status of the PLC, the morphology of the injury, age of the patient, and the neurologic status of the patient are also important determinants. Owing to a high false positive rate, MRI should not be used in isolation to determine the PLC status of the patient.

\section{Conflict of Interest}

No potential conflict of interest relevant to this article was reported.

\section{References}

1. White AA, Panjabi MM. Clinical biomechanics of the spine. Philadelphia (PA): Lippincott; 1978.

2. Vaccaro AR, Lehman RA Jr, Hurlbert RJ, et al. A new classification of thoracolumbar injuries: the importance of injury morphology, the integrity of the posterior ligamentous complex, and neurologic status. 
Spine (Phila Pa 1976) 2005;30:2325-33.

3. Lee HM, Kim HS, Kim DJ, Suk KS, Park JO, Kim NH. Reliability of magnetic resonance imaging in detecting posterior ligament complex injury in thoracolumbar spinal fractures. Spine (Phila Pa 1976) 2000;25:2079-84.

4. Holdsworth F. Fractures, dislocations, and fracturedislocations of the spine. J Bone Joint Surg Am 1970;52:1534-51.

5. Vaccaro AR. Validation of a novel thoracolumbar injury classification and severity score. Chicago (IL): Northwestern University Department of Orthopaedics Grand Rounds; 2005.

6. Vaccaro AR, Zeiller SC, Hulbert RJ, et al. The thoracolumbar injury severity score: a proposed treatment algorithm. J Spinal Disord Tech 2005;18:209-15.

7. Terk MR, Hume-Neal M, Fraipont M, Ahmadi J, Colletti PM. Injury of the posterior ligament complex in patients with acute spinal trauma: evaluation by MR imaging. AJR Am J Roentgenol 1997;168:1481-6.

8. Holdsworth FW. Fractures, dislocations, and fracture-dislocations of the spine. J Bone Joint Surg Br 1963;45:6-20.

9. Nagel DA, Koogle TA, Piziali RL, Perkash I. Stability of the upper lumbar spine following progressive disruptions and the application of individual internal and external fixation devices. J Bone Joint Surg Am 1981;63:62-70.
10. Magerl F, Aebi M, Gertzbein SD, Harms J, Nazarian S. A comprehensive classification of thoracic and lumbar injuries. Eur Spine J 1994;3:184-201.

11. Vaccaro AR, Rihn JA, Saravanja D, et al. Injury of the posterior ligamentous complex of the thoracolumbar spine: a prospective evaluation of the diagnostic accuracy of magnetic resonance imaging. Spine (Phila Pa 1976) 2009;34:E841-7.

12. James KS, Wenger KH, Schlegel JD, Dunn HK. Biomechanical evaluation of the stability of thoracolumbar burst fractures. Spine (Phila Pa 1976) 1994;19:1731-40.

13. Oxland TR, Panjabi MM, Southern EP, Duranceau JS. An anatomic basis for spinal instability: a porcine trauma model. J Orthop Res 1991;9:452-62.

14. Daffner RH, Deeb ZL, Goldberg AL, Kandabarow A, Rothfus WE. The radiologic assessment of post-traumatic vertebral stability. Skeletal Radiol 1990;19:1038.

15. Harris MB, Stelly MV, Villarraga ML, Schroeder AC, Thomas KA. Modeling of the naked facet sign in the thoracolumbar spine. J Spinal Disord 2001;14:252-8.

16. Haba H, Taneichi H, Kotani Y, et al. Diagnostic accuracy of magnetic resonance imaging for detecting posterior ligamentous complex injury associated with thoracic and lumbar fractures. J Neurosurg 2003;99:20-6. 